\title{
Les désaccords sur le changement climatique en France : au-delà d'un climat bipolaire
}

\author{
Hélène Guillemot \\ Histoire et sociologie des sciences, CNRS, UMR 8560, Centre Alexandre Koyré, 75013 Paris
}

\begin{abstract}
Depuis trente ans, les controverses sur la question du climat se sont déployées à la fois dans le débat public et dans les communautés de recherche dans un jeu d'oppositions très réducteur. Le paysage des controverses s'est profondément modifié au gré de négociations chaotiques entre les États et à la faveur de notions émergentes, comme l'adaptation ou d'une prise en compte croissante des questions éthiques. Dans le cadre des Sciences studies, il est proposé ici une cartographie fine des débats scientifiques où il apparaît que les controverses relèvent également des rapports entre les sciences du climat, avec leurs objets et méthodes fondateurs, et les «sciences des impacts du changement climatique " (écologie, hydrologie, géographie, etc.). Entre les critiques portant sur leurs méthodes, alimentant le climato-scepticisme, et celles interrogeant leur utilité pour analyser les impacts et les stratégies d'adaptation qui en découlent, les sciences du climat occupent une situation inédite, originale... et riche de débats futurs.
\end{abstract}

La Rédaction

\section{Mots-clés :}

environnement ;

changement

climatique ;

controverses ; sciences

du climat ;

climato-sceptiques

\section{Keywords:}

environment; climate

change; controversies ;

climate sciences;

climate skeptics
Résumé - Les controverses du climat en France sont souvent réduites à la contestation, par quelques personnalités médiatiques, de la responsabilité humaine du changement climatique. Or cette bipolarisation occulte une plus grande variété de problèmes, d'acteurs et de positions - au-delà de la confrontation entre pro- et anti-environnementalistes à laquelle on l'assimile souvent. Les désaccords portent en particulier sur les sciences du climat et la modélisation - leur légitimité et leurs limites - sur le lien entre science et politique, et sur le cadrage même du problème climatique.

\begin{abstract}
Disagreements on climate change in France: stepping beyond a polarized ambience. Climate change controversies in France are often reduced to popular scientists denying human responsibility in global warming. Such polarization overshadows, however, a wide range of problems, actors and positions. In this paper, we show first that the visibility of these controversies is closely linked to the evolution of the climate issue as a public problem, which has moved through several stages over the past twenty years. The most recent period is characterized by an increase in the diversity of controversies and of "dissonant voices". Part of the disagreements are about climate sciences (especially climate models) and involve the recognition and stabilization of a new disciplinary field and new relationships between science and politics. Another kind of controversy concerns the domain and boundaries of climate sciences, as climate change issues reshape neighbouring scientific fields and confront different epistemic cultures. Finally, political and philosophical disagreements sparked by anthropogenic climate change go beyond the usual conflict between pro and anti- environmentalism, and also concern the very definition of the climate issue.
\end{abstract}

\section{Introduction}

Dans les querelles sur le changement climatique, tout le monde semble au moins d'accord sur le champ de bataille : c'est sur le terrain scientifique que le combat doit être mené. Mais chacun soupçonne l'adversaire de dissimuler des motivations politiques sous des arguments (pseudo) scientifiques. L'objet du conflit, c'est l'origine humaine du changement climatique, dit-on;

Auteur correspondant : helene.guillemot@cnrs.fr 
mais son véritable enjeu est politique, ajoute-t-on. Si les " climato-sceptiques » ne croient pas au changement climatique anthropogénique (ci-après CCA), ce serait $\mathrm{d}^{\prime}$ abord parce qu'ils refusent les changements politiques qu'imposerait la reconnaissance de la responsabilité de l'homme. Les climato-sceptiques, quant à eux, accusent les sciences du climat d'être portées par une idéologie environnementaliste. Dans cet article, nous chercherons à montrer que le tableau n'est pas si simple : même si cette double polarisation est dominante, d'autres désaccords s'expriment à bas bruit, qu'il importe de prendre au sérieux ${ }^{1}$.

Après avoir exposé les difficultés propres à l'étude des controverses du climat, on retracera brièvement la trajectoire du changement climatique comme problème public en France depuis les années 1990. La période récente est caractérisée par une certaine diversification des acteurs et des thèmes de controverses. Une partie des désaccords concerne les sciences du climat, avec la mise en cause des modèles numériques, mettant en jeu la reconnaissance et l'institution d'un nouveau domaine disciplinaire et de nouveaux rapports entre science et politique. Un autre type de controverses questionne le domaine de compétence et les limites des sciences du climat, lorsque la question du changement climatique, de ses causes et de ses impacts reconfigure des domaines scientifiques voisins, faisant se confronter différentes cultures épistémiques. Enfin, en abordant les désaccords politiques et philosophiques suscités par le CCA, on verra qu'ils ne se réduisent pas à un conflit entre pro- et anti-environnementalistes mais visent aussi, notamment, le cadrage du problème climatique. En conclusion, on suggérera que les impasses des politiques climatiques invitent à considérer la variété des débats, plutôt qu'à réaffirmer une polarisation stérile.

\section{Contexte et difficultés de l'étude des controverses sur le climat}

\section{La crainte d'offrir des munitions à l'adversaire}

Si le changement climatique a donné lieu à de nombreux travaux en sciences sociales, les études des sciences ou STS (Science and Technology Studies) ont longtemps été assez peu présentes dans ce domaine ${ }^{2}$. À la fin des années 1990, des sociologues avaient analysé la façon dont des facteurs extra-scientifiques influençaient

\footnotetext{
1 Ce travail bénéficie du soutien du projet ANR ClimaConf.

2 On observe toutefois un renouveau récent. Voir les dossiers sur le changement climatique de Theory, Culture \& Society (marsmai 2010) et Studies in History and Philosophy of Modern Physics (2010, issue 3).
}

les recherches sur le CCA (Shackley et al., 1999 ; Van der Sluijs et al., 1998). Mais leur approche constructiviste a pu sembler s'opposer à un discours s'appuyant sur des vérités scientifiques pour justifier des politiques climatiques (Demeritt, 2001), et ces travaux se sont raréfiés dans les années 2000 : les enjeux politiques tendent à fermer les boîtes noires plutôt qu'à en analyser les contenus. Des raisons similaires expliquent que les controverses du CCA aient été peu étudiées - sauf pour démonter les réseaux climato-sceptiques étasuniens (McCright et Dunlap, 2000 ; Oreskes et Conway, 2010). Le principe méthodologique des analyses de controverses est de décrire symétriquement les acteurs des deux camps, leurs intérêts et leurs argumentations. En permettant ainsi d'exposer la construction complexe des affirmations robustes en même temps que les «bonnes raisons » des vaincus de l'histoire ou des minorités, les études de controverses s'opposent à une vision positiviste ou édifiante de la science, et peuvent être vues comme minant son autorité (Pestre, 2006). Or dans le cas du climat, personne ne veut mettre en cause l'autorité suprême de la science, tous au contraire s'en réclament. À front renversé d'autres controverses sociotechniques, ce sont ici des chercheurs qui ont lancé l'alerte, ceux qui contestent l'expertise du Giec sont aussi des scientifiques - et les écologistes sont les plus fervents supporters de l'expertise officielle.

\section{"Scientifisation » de la politique et politisation de la science}

Le problème du changement climatique se veut conforme à la vision traditionnelle des relations entre science et politique, ou "modèle linéaire », bien résumée par la formule «science speaks truth to power ». Science et politique sont supposées séparées et étanches, ce qui confère sa légitimité à la science comme support de l'action politique. Le Giec revendique cette conception en construisant, à partir des publications scientifiques, un consensus considéré comme nécessaire pour fixer et atteindre des objectifs politiques. Mais le Giec est aussi un agent essentiel de la production d'une vision scientifique globale du climat, en même temps que d'un ordre politique global (Miller, 2004 ; Aykut et Dahan, 2011), et la stricte séparation entre science et politique est ici illusoire (Demeritt, 2001 ; Hulme et Mahony, 2010). Ce décalage entre un discours de purification et des pratiques bien plus hybrides (Latour, 1991 ; Dahan et Guillemot, 2006) a des effets délétères: en faisant peser des exigences irréalistes sur l'expertise du climat, il la rend vulnérable aux désillusions.

Surtout, revendiquer ce mode linéaire de relation entre science et politique aboutit à les rendre encore plus dépendantes l'une de l'autre. En conférant à la science 
un rôle d'arbitre suprême, ce modèle lie le diagnostic et la solution. Des chercheurs ont analysé les mécanismes par lesquels les disputes scientifiques se voient chargées du poids des enjeux politiques (Sarewitz, 2004) : alors $\mathrm{qu}^{\prime}$ on prétend obtenir un accord politique fondé sur un consensus scientifique, on parvient seulement à déguiser des désaccords politiques en dissensus scientifiques (Pielke Jr, 2002). Si l'on suit cette lecture de la politisation du problème, au conflit binaire sur la responsabilité humaine dans le changement climatique se superposerait une bipolarisation politique entre ceux qui prônent une limitation des rejets de $\mathrm{CO}_{2}$ et ceux qui les refusent. On adoptera ici un point de vue légèrement différent, en nous attachant aux désaccords qui ne se rangent pas tout à fait selon cette double polarisation.

\section{Controverses sur les controverses}

Précisons la notion de controverse, car elle est problématique. Aux yeux de beaucoup de climatologues, la grande majorité ${ }^{3}$ des polémiques déclenchées par les climato-sceptiques ne méritent pas le nom de controverses; ce sont des mensonges à dénoncer. Ce type d'objection n'est pas propre à la question climatique : les controverses ont toujours pour enjeu le tracé de frontière entre ce qui est légitime et ce qui ne l'est pas (Gieryn, 1983 ; Jasanoff, 1987). C'est pourquoi la qualification de controverse fait souvent partie de la controverse. Pour contourner cette difficulté, il est commode de distinguer les controverses scientifiques stricto sensu - qui se déroulent suivant des règles établies, entre adversaires se reconnaissant comme légitimes et restent confinées à un public de pairs (Lemieux, 2007) des controverses dites "sociotechniques", beaucoup plus complexes et "impures». Celles-ci débordent dans des arènes publiques, souvent dans les médias, intéressent des groupes variés, mêlent des enjeux et des arguments de différents types (Pestre, 2006) et leur légitimité scientifique est contestée. Ce sont ces controverses, prises au sens le plus large, que nous cherchons à analyser, quels que soient le niveau de désaccord - du débat à la polémique - et les thèmes en jeu.

\footnotetext{
3 Il arrive toutefois (rarement) que les questions soulevées par des sceptiques conduisent à des débats utiles. Ce fut par exemple le cas quand le climatologue Richard Lindzen a mis en cause la capacité des modèles à simuler la rétroaction de la vapeur d'eau ; ou quand a été relevée la contradiction entre des mesures de températures au sol et par satellites; ou encore quand ont été repérées des erreurs statistiques sur les données de la courbe de la température moyenne du globe depuis mille ans. Aujourd'hui closes, ces controverses n'ont pas invalidé la réalité du CCA.
}

Certains objecteront que la vérité scientifique prime, et qu'il est insupportable de mélanger les mensonges des sceptiques et de véritables débats. Mais, on le verra, ce distinguo n'est pas aussi clair dans l'espace public: arguments scientifiques, désaccords politiques et critiques diverses se mêlent souvent. Examiner les désaccords dans leur diversité au lieu de les ranger dans des catégories préétablies permet de déployer la pluralité des enjeux.

\section{Le problème climatique en France : construction, médiatisation et controverses}

\section{La construction du problème public}

La visibilité médiatique des controverses est étroitement liée à l'évolution de la question climatique comme « problème public » (Gusfield, 1981). Dans une étude sur les médias français de 1990 à 20104 , nous avons identifié, avec Stefan Aykut et Jean-Baptiste Comby (Aykut et al., 2012), trois phases distinctes de médiatisation des controverses, qui correspondent à trois étapes dans la trajectoire publique du changement climatique. Pendant les années 1990, le CCA n'est pas encore constitué en problème public en France. Malgré une importante activité diplomatique et administrative, le débat médiatique est faible. Les discours d'opposants sont cependant présents, notamment au moment du Sommet de la Terre à Rio en 1992, lorsque se cristallise, autour de «l'appel de Heidelberg ${ }^{5} »$, une mouvance intellectuelle réagissant contre la montée de l'écologie politique. C'est aux alentours de 2002 ou 2003 que le CCA devient un problème public majeur en France. Il s'institutionnalise ${ }^{6}$ et sa couverture médiatique augmente ; les scientifiques du climat s'expriment davantage, tandis que les sceptiques sont

\footnotetext{
4 Le corpus comprenait une étude de contenu des journaux télévisés de 20 h sur TF1 et France 2, d'articles sur le CCA dans Le Monde, Sud Ouest et L'Express et s'appuyait également sur une centaine d'interviews.

5 L'appel de Heidelberg, signé par près de 4000 scientifiques, dont 72 prix Nobel, s'adressait aux chefs d'État réunis à Rio en 1992 pour le Sommet de la Terre en dénonçant « une idéologie irrationnelle qui s'oppose au progrès scientifique et industriel et entrave le développement économique et social ».

6 Avec notamment la loi de 2001 attribuant la qualité de priorité nationale à la lutte contre l'effet de serre et à la prévention des risques qui lui sont liés, la création de l'Observatoire national sur les effets du réchauffement climatique (Onerc) et la mise en place du Programme de lutte contre le changement climatique.
} 
marginalisés ${ }^{7}$. Quatre groupes participent activement au cadrage de la question climatique : les journalistes scientifiques et d'environnement, les scientifiques du climat, les services d'État chargés de ce problème (par exemple l'Ademe) et les ONG environnementales. Ils ont chacun connu des évolutions importantes au début des années 2000 (ainsi les sciences du climat en France ont vu la mise en place de nouvelles institutions et se sont davantage impliquées dans le Giec). Ces évolutions favorisent la coopération entre ces groupes, qui partagent la conviction que la lutte contre le CCA passe par des changements de comportements individuels. Durant cette deuxième phase, ils vont ensemble définir le CCA comme un problème public qui fait consensus et doit fédérer les citoyens, qu'il s'agit de « sensibiliser » à cette question. Ce cadrage laisse peu de place aux incertitudes et aux controverses.

Pourtant, cette deuxième période n'est pas exempte de disputes médiatiques. Les opposants qui accèdent aux médias cumulent souvent capital scientifique, académique et politique ${ }^{8}$. Contestant la réalité du CCA sans être climatologues, ils sont disqualifiés par ces derniers et par les journalistes spécialisés, comme scientifiquement illégitimes et minoritaires ${ }^{9}$.

\section{Des climato-sceptiques plus visibles mais marginalisés dans les principaux médias}

Une troisième phase dans la carrière du CCA s'est ouverte en 2008-2009. On dit souvent que la question climatique a reculé sous l'assaut d'une vague climato-sceptique coïncidant avec l'échec du sommet de Copenhague et avec les affaires qui ont éclaboussé le Giec ${ }^{10}$. Une étude des médias français de 2007 à mi-2011 invite à

\footnotetext{
7 Les climato-sceptiques restent invisibles dans les 663 sujets sur le climat diffusés dans les JT de 20 h de TF1 et France 2 entre 1997 et 2006 (source Ina, Aykut et al., 2012).

8 Les principales personnalités contestant le CCA ont un profil caractéristique :H. Tazieff, C. Allègreet V. Courtillot sont des géophysiciens occupant des positions académiques importantes et des fonctions politiques gouvernementales.

9 C'est le cas notamment de Claude Allègre dont plusieurs chroniques dans $L^{\prime}$ Express ont fait scandale durant cette période. Son article intitulé « Neiges du Kilimandjaro » (21 septembre 2006) a suscité onze réponses dans Le Monde, Libération et L'Express, de journalistes spécialisés et de scientifiques, ces derniers ayant aussi adressé des lettres à plusieurs institutions telles que l'Académie des sciences et le ministère de la Recherche.

10 À l'automne 2009, le piratage et la publication d'environ mille courriels de climatologues de l'université d'East Anglia (« climategate ») ont été suivis par la révélation d'erreurs dans le $4^{\mathrm{e}}$ rapport du Giec.
}

nuancer cette affirmation (Comby et Romanet, 2012). La médiatisation du CCA a culminé en 2007 (année du $4^{\mathrm{e}}$ rapport du Giec, de l'attribution du prix Nobel de la paix à celui-ci, et en France du Grenelle environnement) ; elle diminue ensuite, malgré une nouvelle hausse en 2009 au moment du sommet de Copenhague. Cette érosion de la couverture médiatique du climat s'explique en partie par la prééminence d'autres actualités, la crise économique et l'échec des négociations. Mais elle est aussi la rançon du succès de la mobilisation : le CCA est devenu un problème public majeur, pris en charge au plus haut niveau de l'État - avec la création d'un grand ministère de l'Environnement, disposant de moyens inédits - et la médiatisation devient moins essentielle. Le changement climatique est devenu un «lieu commun» des débats publics, un thème central du monde contemporain. Il est moins médiatisé pour lui-même, mais on l'évoque dans de nombreux domaines.

D'autre part, l'étude montre que si les articles sur le CCA ont diminué en volume, leur traitement a peu changé et le cadrage de la phase précédente reste solide $^{11}$. Les sciences du climat et le Giec font toujours autorité, les climatologues restent très consultés par les journalistes, tandis que les climato-sceptiques et leurs idées demeurent marginaux dans les médias généralistes ${ }^{12}$ - grâce notamment à la très forte mobilisation de journalistes spécialisés. Les climato-sceptiques trouvent cependant un meilleur accueil dans des espaces à audience plus réduite et plus cultivée (tribunes de presse écrite, magazines d'actualité, émissions de radio ou débats télévisés ${ }^{13}$ ), animés par des professionnels qui ne partagent pas les mêmes normes que les journalistes

\footnotetext{
${ }^{11}$ Dans d'autres pays, le cadrage du changement climatique semble avoir davantage évolué à la suite de la conférence de Copenhague et du «climategate »-comme au Royaume-Uni ; voir l'article du climatologue Mike Hulme: http:// www.theguardian.com/environment/2010/nov/15/year-climate-science-was-redefined.

12 De janvier 2009 à août 2011, sur 1423 articles du Monde sur le changement climatique, une trentaine rend visibles les climato-sceptiques ou leurs arguments. De janvier 2007 à aout 2011, sur 173 articles de L'Express, huit les mentionnent. Pendant la même période, aucun climato-sceptique n'apparaît dans les 311 reportages des JT de 20 heures de TF1 ; sur France 2, deux reportages sur 321 font état de controverses (Comby et Romanet, 2012).

13 On s'inquiète souvent de l'impact sur le public des sites climato-sceptiques sur Internet. Il faut relativiser leur rôle pour trois raisons: les sites environnementalistes et défendant la cause climatique sont bien plus nombreux sur la Toile ; les prestiges des sites sont très inégaux, et Internet reflète plus qu'il ne bouscule les rapports de force extérieurs ; enfin, l'exposition à l'information est très sélective: les gens consultent des sites dont les sujets les intéressent, et qui professent des opinions proches des leurs (Comby et Romanet, 2012).
} 
scientifiques ${ }^{14}$. Cette visibilité médiatique, bien qu'assez circonscrite, est inhabituelle et a pu choquer ou inquiéter en particulier ceux qui ont contribué au cadrage du problème (on reviendra plus loin sur les ressorts de cette inquiétude dans le cas des scientifiques du climat).

\section{Diversité des thèmes et des acteurs de controverses}

Un autre fait marquant, bien moins souligné que la recrudescence climato-sceptique, est la diversification des thèmes de controverses et l'apparition de nouveaux acteurs. Pour en rendre compte, nous distinguerons trois groupes exprimant des «voix dissonantes ${ }^{15}$ ». Le premier groupe est celui des climato-sceptiques déjà présents dans la période précédente. Dans cette troisième phase (plus particulièrement de juin 2009 à juin 2010), ils apparaissent plus souvent dans certains médias comme on vient de le voir, surtout à l'occasion de la publication $\mathrm{d}^{\prime}$ ouvrages ${ }^{16}$, auxquels des climatologues et des journalistes ripostent par des livres qui en dénoncent les erreurs et les mensonges ${ }^{17}$. Mais d'autres acteurs font maintenant entendre leurs désaccords sans forcément remettre en cause la réalité du CCA : des scientifiques dont le domaine d'étude est concerné par le changement climatique, notamment par ses impacts - qu'on désignera ici comme "groupe $2 »^{18}$ - et aussi des intellectuels non scientifiques qui émettent des désaccords d'ordre politique ou éthique - qui constituent notre « groupe $3 »^{19}$.

Avant de développer la variété des disputes sur le climat dans les prochaines sections, et pour clore ce bref

14 Les journalistes spécialisés, qui jouent un rôle majeur dans le cadrage de la question climatique, doivent souvent lutter pour imposer ce cadrage dans leurs propres médias. Plusieurs formes de légitimité se confrontent en effet au sein d'une rédaction - les journalistes scientifiques ou d'environnement défendant les critères de légitimité scientifique, d'autres professionnels pouvant privilégier des critères plus politiques.

15 Nous employons ce terme pour désigner des acteurs qui expriment des désaccords sans nécessairement s'opposer à la réalité du CCA.

16 En particulier Nouveau Voyage au centre de la Terre, de V. Courtillot en septembre 2009; L'imposture climatique : ou la fausse écologie de C. Allègre en février 2010 ; Le Mythe climatique de B. Rittaud en février 2010.

17 En particulier L'imposteur, c'est lui. Réponse à Claude Allègre, de S. Huet en avril 2010 ; Le Populisme climatique de S. Foucart (journaliste scientifique au Monde) en octobre 2010 ; Climat : le vrai et le faux, de V. Masson-Delmotte (climatologue) en février 2011.

18 Ce groupe compte notamment des géographes (Sylvie Brunel, Jean-Robert Pitte, Martine Tabeaud, etc.) et le démographe Hervé Le Bras.

19 Le philosophe Dominique Lecourt, le politiste François Ewald, le sociologue Jean-Pierre Le Goff, l'écrivain Pascal Bruckner, le journaliste Olivier Postel-Vinay, etc. survol de deux décennies de controverses sur le climat, il faut rappeler les différences essentielles entre les situations américaine et française. Alors qu'aux États-Unis le CCA est très clivant politiquement, en France le consensus s'étend sur tout l'échiquier politique pour admettre la réalité du problème. Après le refus américain de ratifier le protocole de Kyoto en 2000, la lutte contre le changement climatique a constitué un pilier de la politique européenne ; en France, il a été inséré dans des politiques publiques aux niveaux national et territorial. Les médias français, à la différence des médias américains, donnent très majoritairement du CCA une présentation consensuelle, et non conflictuelle. Enfin, les climato-sceptiques et les « voix dissonantes » s'expriment individuellement et ne sont pas organisés dans des collectifs ; et ils ne sont pas liés financièrement à l'industrie pétrolière. Si le point commun des climato-sceptiques américains est le rejet de toute contrainte sur les émissions de gaz à effet de serre, il n'en va pas de même pour leurs homologues français. Ces différences interrogent les spécificités des disputes sur le climat en France.

\section{Les sciences du climat dans la bataille}

\section{Les modèles numériques sur la sellette}

Les désaccords sur le climat, dans cette troisième phase surtout, sont variés et mêlent des arguments de registres différents. Cela vaut aussi pour le groupe 1 , celui des climato-sceptiques, qui sont tous des scientifiques de disciplines éloignées du climat. Au centre de leurs livres et articles, plus que le changement climatique lui-même, ce sont souvent les sciences du climat qui sont attaquées. Les climatologues sont accusés de fonder leurs affirmations sur des modèles peu fiables, incomplets et mal validés par les données. Les données d'observation sont aussi sur la sellette, mais c'est la critique des modèles numériques du climat qui constitue un "point épistémologique central » pour les climatosceptiques français ${ }^{20}$. En cela, ils rejoignent leurs homologues américains, qui «cherchent à stigmatiser la modélisation comme science inférieure sur des bases philosophiques » (Norton et Suppe, 2001) en l'opposant

\footnotetext{
20 C'est ce qu'affirme Claude Allègre dans une tribune du Monde du 22 mai 2010 intitulé « Le droit au doute. On ne peut pas modéliser un système naturel complexe en alignant des équations ». Outre C. Allègre et V. Courtillot, d'autres attaquent les modèles de climat : le physicien S. Galam ( Pas de certitude scientifique sur le climat », Le Monde, 7 février 2007), le biologiste H. Atlan («La religion des catastrophes », Le Monde, 27 mars 2010) et le mathématicien B. Rittaud, déjà cité.
} 
à la sound science fondée sur des " théories assurées » et des « données brutes » (Edwards, 1999).

$\mathrm{Si}$ ces attaques peuvent avoir quelques effets auprès de certains publics, scientifiques notamment, c'est parce que les sciences du climat, mobilisant des disciplines variées, mêlant modélisation, théorisation, simulations et analyses des données, relèvent d'une épistémologie différente des sciences théorico-expérimentales classiques (Stengers, 1993). Les modèles englobent des théories physiques, mais aussi des représentations simplifiées de processus complexes - les «paramétrisations »- qui sont moins solidement étayées (même si elles donnent lieu à des recherches très approfondies). Les validations sont souvent très indirectes, et supposent des allers et retours entre observations et simulations. Quant aux projections du climat futur, elles ne peuvent évidemment pas être directement comparées à des observations... mais on peut en contrôler certains résultats par des méthodologies subtiles (Guillemot, 2010). Résultant de cette pluralité et de cette hétérogénéité méthodologique, les modèles fournissent à la fois des prédictions considérées comme pratiquement certaines (le réchauffement global, sa répartition à grande échelle) et d'autres, entachées d'incertitudes parfois considérables (les précipitations dans certaines régions du globe).

Il y a donc une vraie tension entre la nouveauté épistémique de ces modélisations et leur rôle central dans l'expertise du Giec, au cœur des négociations climatiques. Les sciences du climat constituent l'exemple même de ce que Funtowicz et Ravetz (1993) appellent des sciences «post-normales », caractérisées par «des faits incertains, des valeurs discutées, des enjeux élevés et des décisions urgentes ». Même si les critiques de la modélisation paraissent grossières, il serait donc trop simple de les réduire à de purs prétextes politiques. La méfiance $\mathrm{qu}^{\prime}$ on rencontre chez certains physiciens, mathématiciens ou ingénieurs relève, pour partie, de conflits de «cultures épistémiques »-des scientifiques ayant des pratiques et des méthodologies éloignées développent des épistémologies et des conceptions de la science différentes (Knorr-Cetina, 1999). Comme l'écrit un climatologue, " paramétrisation, tuning et debugging laissent sceptique une frange de la population scientifique, en particulier les ingénieurs, habitués à des modèles asymptotiquement exacts » (Crucifix, 2011).

Les composantes épistémiques et politiques de cette méfiance ne sont pas exclusives, elles tendent au contraire à se renforcer mutuellement. Une anthropologue a montré la dimension culturelle de l'engagement de physiciens sceptiques aux États-Unis : ils défendent certes des valeurs politiques et leur situation d'élites intellectuelles menacées par la montée des questions environnementales, mais aussi des visions normatives de la science et de la modernité ancrées dans leurs propres pratiques disciplinaires (Lahsen, 2008). On reviendra plus loin sur ce rapport entre critiques épistémique et politique.

\section{Mise en cause de la légitimité scientifique}

Certaines attaques climato-sceptiques vont plus loin que la critique de la modélisation, et visent la légitimité des sciences du climat. Ainsi, dans son livre à succès L'imposture climatique, Claude Allègre accuse les climatologues de manque de rigueur, voire de tricherie et de complot. C'est pour répondre à ces violentes attaques que 600 scientifiques du climat ont adressé, en avril 2010, une lettre ouverte à leurs tutelles leur demandant de reconnaître "l'intégrité et le sérieux » de leur travail ${ }^{21}$. Par cette pétition (parfois mal comprise ${ }^{22}$ ), ils répondaient à Allègre sur le terrain qu'il avait choisi : non pas celui du débat scientifique mais celui de la légitimité et de l'honnêteté professionnelle. D'où l'appel à l'autorité ministérielle, habilitée à dire la science légitime, et non la vérité scientifique. On pourrait s'étonner que les chercheurs d'un domaine aussi important et influent réclament ce soutien institutionnel : ce serait méconnaître le sentiment de manque de reconnaissance répandu dans la communauté. Cette vulnérabilité a plusieurs origines, certaines liées à des spécificités françaises, d'autres plus fondamentales.

On ne s'étendra pas sur les rivalités des climatologues avec leurs voisins de l'Institut de physique du globe de Paris à Jussieu, dirigé par Allègre puis par Courtillot, ni sur les nombreux soutiens dont bénéficient ces derniers, notamment à l'Académie des sciences. Un autre facteur de vulnérabilité est au moins aussi important : les scientifiques du climat déplorent depuis longtemps l'absence d'implication de leurs tutelles dans le domaine du changement climatique. Le problème du climat a d'abord été institutionnalisé au niveau international ; en France, sa prise en charge a été plus tardive et moins structurée qu'ailleurs. Ainsi, contrairement à leurs collègues d'autres pays, les climatologues français n'ont reçu

\footnotetext{
21 À la suite de cette lettre ouverte adressée à la ministre de la Recherche, au président de l'Académie des sciences ainsi qu'aux directeurs du CNRS et de grands instituts de recherche français (CEA, Inra, etc.), la ministre de la Recherche a demandé à l'Académie des sciences l'organisation d'un débat - qui s'est tenu le 20 septembre 2010 et a donné lieu à un rapport réaffirmant le consensus scientifique - et la direction du CNRS a mis en place une mission de réflexion et d'enquête, qui a abouti à la publication en 2011 d'un ouvrage (Le climat à découvert, éditions du CNRS) exposant les méthodes et outils des sciences du climat.

${ }^{22}$ Le physicien et épistémologue J.-M. Lévy-Leblond écrit ainsi : «Que les signataires jugent nécessaire de faire appel aux pouvoirs politiques et administratifs pour conforter leurs positions revient à reconnaître le manque d'indépendance qui leur est reproché par leurs adversaires, et qui obère la fiabilité des travaux du Giec » (« Le scientifique, le climat et le politique », Libération du 8 avril 2010).
} 
aucune incitation à participer aux simulations pour le Giec de la part des politiques. Le manque d'articulation entre les chercheurs travaillant sur le changement climatique et les responsables ministériels chargés de ce problème a été également manifeste lors de négociations internationales ${ }^{23}$. La lettre ouverte des climatologues $\mathrm{s}^{\prime}$ inscrit dans cette histoire marquée à la fois par la faiblesse des relations entre les scientifiques français du climat et leurs tutelles politiques, et par la puissance des réseaux d'influence des hiérarques de disciplines rivales.

Au-delà du cas français, cette fragilité ressentie par les chercheurs est aussi liée à la position et à l'exposition de la science du climat, " universelle, hégémonique et cependant faiblement enracinée » (Jasannoff et Wynne, 1998). Soumise à des contraintes d'expertise très lourdes, exposée médiatiquement, elle est confrontée à des adversaires pugnaces... et à des alliés parfois encombrants. Les climatologues, un peu « mal à l'aise » face aux écologistes se réclamant de « la science ${ }^{24}$, entendent à la fois montrer que «leur communauté fonctionne avec la même rigueur et les mêmes critères que les autres domaines scientifiques, comme la physique, la chimie, etc. » et combattre « l'instrumentalisation » et la " confusion entre chercheurs, écologistes et politiques $»^{25}$. Si les attaques climato-sceptiques les ont inquiétés, c'est moins pour l'impact médiatique de la négation du CCA, qu'à cause du soupçon porté sur leur discipline, sa scientificité et à son impartialité, qui risque de miner la confiance et la reconnaissance des autres communautés scientifiques. Pour les scientifiques du climat, le double «travail de frontières » (Gieryn, 1983), pour se démarquer du politique comme pour instituer et faire reconnaître leur domaine disciplinaire, constitue un enjeu majeur ${ }^{26}$.

${ }^{23}$ Plusieurs scientifiques français déplorent ce "déficit de liens » entre scientifiques et politiques (interviews de $\mathrm{H}$. Le Treut, J. Jouzel, S. Joussaume, O. Boucher, J.-C. Hourcade in Guillemot, 2007).

${ }^{24}$ Quand les climatologues français se sont engagés dans l'expertise du Giec, ils ont parfois collaboré avec des ONG environnementalistes (Greenpeace, Réseau action climat) qui " remplissaient un vide » laissé par l'absence des tutelles institutionnelles. Depuis 2010, les scientifiques du climat prennent à nouveau leurs distances : bien que reconnaissant le rôle de ces ONG, ils se sentent souvent « mal à l'aise » devant les discours se réclamant de l'autorité de «la science » (interview de $\mathrm{H}$. Le Treut par C. Buffet, mai 2010).

25 Édouard Bard et Valérie Masson-Delmotte, « Pédagogie de la complexité plutôt que démagogie de la simplicité. Les responsables de la pétition contre $\mathrm{M}$. Allègre expliquent leur initiative », Le Monde, 22 mai 2010.

26 L'importance de cette reconnaissance institutionnelle est soulignée par Bruno Latour (2012) lorsqu'il rapporte, en introduction de son dernier ouvrage, la réaction a priori surprenante d'un climatologue qui, pour répondre à un industriel doutant du changement climatique, en appelle non pas à la vérité scientifique mais à la confiance dans l'institution.

\section{Extension du domaine du climat}

\section{Conflits aux frontières}

Il est un autre type de controverses, moins violentes, qui ne visent pas le cœur des sciences du climat et ne remettent pas en cause leur légitimité, mais en questionnent les limites, le domaine de pertinence. Alors que les attaques des modèles sont le fait de scientifiques sans lien avec le climat (groupe 1), ces controverses proviennent de chercheurs de disciplines concernées par les impacts du CCA (groupe 2). De nombreux secteurs scientifiques sont en effet touchés, qu'il s'agisse d'inclure de nouveaux processus dans les modèles (des modules d'hydrologie ou d'évolution des écosystèmes par exemple), d'évaluer les conséquences des changements climatiques (sur l'agriculture, la santé) ou d'envisager des mesures d'adaptation. Cette expansion de l'aire d'influence du climat ne va pas sans heurts. L'impératif de modélisation, la démarche descendante, la primauté $\mathrm{du}$ facteur climatique ne sont pas toujours jugés pertinents par les chercheurs qui travaillent à d'autres échelles, à partir d'autres paradigmes.

La traduction locale du CCA, en particulier, soulève plusieurs difficultés. Le changement climatique a d'abord été envisagé comme une question d'équilibre radiatif global : les évolutions climatiques sont modélisées à grande échelle, puis, par descente d'échelle ou par d'autres techniques comme la «désagrégation statistique », sont calculées les manifestations locales du réchauffement et ses impacts dans différents domaines. L'échelle globale, la mieux maîtrisée scientifiquement, est aussi celle qui offre le moins de prise sensible. La demande politique porte de plus en plus sur le niveau régional, mais à mesure que l'échelle diminue, les mécanismes climatiques sont moins bien compris, les incertitudes augmentent; et le problème du CCA, quand on cherche à l'ancrer localement, semble se dissoudre au milieu de nombreuses questions tout aussi majeures traitées dans d'autres cadres par d'autres sciences. Pour certains géographes par exemple, qui étudient sur le terrain les interactions entre milieux naturels et sociétés humaines, la primauté du climat global ne va pas de soi. Il y a donc, là encore, une dimension scientifique et épistémique dans certaines critiques (même si, à nouveau, on ne peut ignorer que s'y mêlent parfois des considérations politiques ${ }^{27}$ ). Mais la difficulté dépasse la question

\footnotetext{
27 Les géographes, en France, sont divisés sur le problème. La Société de géographie a organisé en septembre 2010 un colloque intitulé " Non, le ciel ne va pas nous tomber sur la tête", d'où est issu un livre éponyme, et a remis son Grand Prix à Claude Allègre pour son livre L'Imposture climatique. En partie en réaction contre cette position, un colloque international s'est tenu en septembre 2012 sur le thème " Géographie, écologie, politique : un climat de changement » pour questionner "sans instrumentalisation politique » les réticences de la géographie française à aborder les questions écologiques.
} 
académique des frontières disciplinaires; plus fondamentalement, le CCA peine à « relier les grandes échelles de la représentation scientifique aux plus petites échelles de la signification sociale » (Jasanoff, 2010). Par exemple, les critiques de la température moyenne du globe comme indicateur central du changement climatique peuvent toucher juste (au-delà des subtilités scientifiques liées à cette notion), en pointant le problème essentiel de la traduction locale d'un phénomène d'abord construit comme global.

\section{Les impacts du changement climatique... sur les sciences voisines}

Loin de confondre tous les "sceptiques ", les climatologues sont souvent sensibles aux objections de collègues dont les disciplines sont concernées, voire reconfigurées par les effets du CCA ${ }^{28}$. D'autant que la question des limites des modèles se pose avec force d'abord aux modélisateurs eux-mêmes. La fiabilité des prévisions régionales, la pertinence des prévisions décennales, ou l'utilisation des modèles pour l'adaptation constituent des sujets de recherche très vivaces qui font débat dans la communauté des sciences du climat (Dessai et al., 2009). Certains climatologues sont convaincus de la nécessité et de la faisabilité des prévisions aux courtes échelles spatiales ou même temporelles. D'autres considèrent au contraire que les modèles ne sont pas capables de fournir des prévisions fiables et utiles à ces échelles (Trenberth, 2010) - ou que la variabilité naturelle du climat compromet parfois la pertinence même des prévisions régionales (Deser et al., 2012).

Le vaste domaine des impacts du CCA connaît aussi des controverses, en particulier quand on attribue au climat des phénomènes qui relevaient d'autres causalités. $C^{\prime}$ est le cas pour la question sensible des réfugiés climatiques ; ainsi le démographe Hervé Le Bras (signataire de l'appel de Heidelberg) a sévèrement critiqué l'évaluation des 300000 victimes du changement climatique ${ }^{29}$. De même, certaines questions de développement ont été reconfigurées par la focalisation sur les stratégies d'adaptation au CCA. Attribuer des catastrophes au réchauffement global peut reléguer au second plan des paramètres socioéconomiques étudiés par d'autres

\footnotetext{
${ }^{28} \mathrm{H}$. Le Treut (climatologue modélisateur, directeur de l'Institut Pierre-Simon Laplace) écrit ainsi : «Iln'y a pas deux camps, mais tout un dégradé d'opinions. Le climato-scepticisme touche [...] des géographes, des géologues, des hydrologues, des écologues, des économistes... Ils ont beaucoup de choses à nous dire, car la complexité ou l'incertitude dont ils parlent joue effectivement un rôle important » (in Chappellaz et al., 2010).

29 «300 000 morts climatiques » par H. Le Bras, La Recherche, septembre 2009.
}

scientifiques (Cannon et Müller-Mahn, 2010 ; Mercer, 2010) ou des causes de vulnérabilité mises en avant par les populations autochtones pour qui le climat n'est pas le facteur essentiel. On rejoint des arguments d'une «écologie politique critique » qui analyse les conséquences politiques de l'influence grandissante des sciences de l'environnement dans les pays en développement (Forsyth, 2003).

\section{Anti-environnementalisme et autres désaccords}

\section{Une relation singulière entre science, politique, raison et État}

Le troisième groupe de « voix dissonantes » aborde des questions politiques, éthiques ou philosophiques. Les aspects politiques sont déjà très présents dans les controverses des groupes 1 et 2, on l'a vu, et se mêlent souvent aux considérations scientifiques. Pour autant ces désaccords ne sont pas réductibles à des batailles politiques. Même si la bipolarisation domine le champ, on n'a pas d'un côté la pure vérité scientifique et de l'autre les seules passions politiques.

On a parfois relevé la filiation des positions climatosceptiques avec la mouvance anti-environnementale qui s'est notamment manifestée il y a vingt ans autour de l'appel de Heidelberg (Millier, 2011). Les climatosceptiques français poursuivraient ce combat contre l'écologie et pour la défense de valeurs scientistes et productivistes ; ainsi, Claude Allègre cherche depuis plus de vingt ans à promouvoir une " autre vision de l'écologie », une "écologie de la raison » fondée sur les solutions scientifiques ${ }^{30}$. Ce combat politique va de pair avec une certaine vision de la science, liée aux valeurs d'innovation, d'émancipation et de progrès. Or, les nouvelles disciplines qui alertent sur les effets des activités humaines sur l'environnement et la santé, conduisent à remettre en cause les bienfaits des avancées technoscientifiques, et bousculent la relation privilégiée nouée entre science et politique, en France tout particulièrement ${ }^{31}$. Les spécificités du climato-scepticisme à la française doivent sans doute beaucoup à cette relation singulière entre science, politique, raison et État, enracinée

\footnotetext{
30 Tel est aussi le credo de la fondation Écologie d'avenir créée par C. Allègre en 2010 sous l'égide de l'Institut. Son conseil d'orientation accueille de nombreuses personnalités du monde intellectuel, des sciences et de l'industrie, dont trois prix Nobel.

31 Ce lien singulier est personnifié par la figure du haut fonctionnaire issu d'un grand corps de l'État, dont la double légitimité scientifique et politique assure la capacité de représenter tout à la fois la vérité et l'intérêt général du pays.
} 
dans l'histoire de la République ${ }^{32}$. Mais là encore, il serait trop simple de réduire les critiques d'ordre politique, éthique ou philosophique du changement climatique à la bataille entre partisans de l'écologie et thuriféraires du progrès.

Les voix dissonantes sont individuelles, non organisées, animées par des motivations diverses. Les acteurs du groupe 3 ne peuvent être rassemblés sous une étiquette idéologique (productiviste, rationaliste, favorable au capitalisme, etc.). Ainsi deux des intellectuels ayant exprimé des désaccords sur la question climatique, Dominique Lecourt ${ }^{33}$ et Jean-Marc Lévy-Leblond ${ }^{34}$ se sont fortement prononcés contre l'appel de Heidelberg il y a vingt ans (avec des perspectives d'ailleurs différentes) [Lecourt, 1993 ; Lévy-Leblond, 1996]. À l'inverse, le cancérologue et académicien Maurice Tubiana, qui fut l'un des premiers signataires de cet appel et un grand contempteur du principe de précaution, faisait partie de l'association Sauvons le climat, qui défend la cause du CCA à partir d'une position favorable à l'énergie nucléaire. L'Union rationaliste, défenseur des valeurs de rationalisme et de progrès et plutôt hostile aux idées écologistes, soutient la lutte contre le $\mathrm{CCA}^{35}$. On pourrait multiplier les exemples.

Assimiler les voix dissonantes à un camp sceptique, même en reconnaissant la diversité des motivations (Godard, 2012), renforce la polarisation et laisse dans l'ombre d'autres enjeux. Plutôt que figer les positions pour ensuite s'étonner de ces "alliances improbables » (Foucart, 2010), on pourrait être attentif à la façon dont le problème du climat fait évoluer les causes et bouger les frontières, dont il prend des significations différentes selon qui s'en empare.

\section{Critiques du scientisme, critiques du cadrage}

D'autres lignes de fractures, moins visibles, doivent être prises en compte pour comprendre certains points de vue critiques. Il est en particulier des désaccords dont on parle peu : ceux qui touchent au scientisme - et plus généralement, au cadrage du problème climatique. Et

\footnotetext{
32 Pour Bruno Latour, Claude Allègre, en montant au créneau contre ces sciences nouvelles, incarnerait le rêve français d'une "République fondée en raison » ("Quand le principe de précaution déstabilise le rationalisme à la française ", Le Monde, 22 mai 2010).

33 D. Lecourt est aussi membre du conseil d'orientation de la fondation Écologie d'avenir fondée par C. Allègre.

34 J.-M. Lévy-Leblond dirige la collection Science ouverte au Seuil, où est publié Le Mythe climatique de B. Rittaud.

35 Le vice-président de l'Union rationaliste, Michel Petit, est l'un de premiers chercheurs français ayant participé au Giec.
}

pour cause: la conviction selon laquelle la science est l'unique autorité qui justifie la décision politique (Wynne, 2010) est partagée par les climato-sceptiques du groupe 1 comme par les défenseurs de la cause climatique (ils diffèrent évidemment sur ce qu'est la bonne science). Cette place de la science est cependant contestée par d'autres, notamment du groupe 3, qui épinglent un positivisme d'obédience écologiste - tels l'écrivain Pascal Bruckner ${ }^{36}$ et Dominique Lecourt, qui dénonce «ce mode de pensée positiviste» qui «subordonne l'action à la connaissance ${ }^{37}$.

Là encore, les registres critiques se mêlent. On peut d'un même mouvement (comme D. Lecourt) s'opposer à une conception politique, suspecter la science censée la justifier et réprouver le rapport d'autorité qui les relie. Mais on peut aussi critiquer le régime climatique d'un point de vue différent, comme le sociologue des sciences Brian Wynne (2010) pour qui le scientisme contribue à la vulnérabilité des sciences du climat: «Le succès des sceptiques repose sur la façon même dont est posée la question politique, avec la science placée au centre [...] Paradoxalement, cela se produit quand la science atteint sa plus grande influence politique, qu'elle ne se contente pas de produire des faits mais qu'elle définit le sens politique des problèmes. »

De nombreux aspects du régime climatique et de son cadrage sont critiqués : le mode de négociations onusien, le marché du carbone, une certaine hégémonie de la question climatique, au détriment d'autres problèmes jugés plus importants. Le sociologue Jean-Pierre Le Goff dénonce une «vision naturaliste et moralisante » du problème climatique qui en « dissout la dimension culturelle et politique ${ }^{38}$. La présentation du CCA comme " cause humanitaire globale » conduit en effet à évacuer les divergences, à le dépolitiser (Swyngedouw, 2010). Définir le CCA comme l'affaire de tous permet de valoriser l'enjeu comme universel, mais aussi de ne pas mettre en cause les intérêts de certains secteurs économiques ou catégories sociales, et d'orienter les solutions vers des choix individuels plutôt que vers des infrastructures publiques par exemple (Comby et Grossetête, 2012).

Mentionnons enfin une autre critique touchant à une dimension essentielle du CCA : le rapport qu'il instaure à l'avenir. Le géographe climatologue Mike Hulme (2011) fustige le « réductionnisme climatique » résultant

\footnotetext{
36 «Le réchauffement qui refroidit », par P. Bruckner, Libération, 13 janvier 2010.

37 «La guerre du climat », par D. Lecourt, La Croix, 30 mars 2010.

38 «La naissance du bobo rousseauiste », par J.-P. Le Goff, Marianne 2, 12 juin 2009.
} 
du rôle central des modèles globaux dans le problème du $\mathrm{CCA}^{39}$. Le climat, seul facteur (très partiellement) connaissable du futur grâce à la modélisation physique, en devient la variable de prédiction dominante. En occultant la complexité des interactions entre éléments humains, sociaux et naturels, cette conception marginalise les autres facteurs contribuant à façonner l'avenir. On rejoint là une critique de la rhétorique de la catastrophe et de l'urgence, à l'œuvre dans nombre de discours sur le climat ${ }^{40}$.

\section{Conclusion}

Dans cet article, on a voulu montrer que les controverses du climat sont bien plus riches et diverses que la seule question de la réalité du changement climatique d'origine humaine. Les sciences sont au centre de nombre de ces débats : sont en jeu la fiabilité des modèles de climat, le périmètre de leur utilisation, mais aussi la reconnaissance d'un mode de production des savoirs, l'institution d'un domaine disciplinaire et ses limites ; et encore la légitimité et l'autorité de ces sciences, leur rapport spécifique à l'expertise et au pouvoir. Mais ces divergences ne s'expriment qu'à bas bruit, aux marges d'un débat polarisé. La confrontation binaire dominante tend en effet à faire taire les désaccords - ou à en laisser le monopole aux climato-sceptiques, qui peuvent ainsi cristalliser des oppositions variées ${ }^{41}$.

La polarisation du débat climatique résulte en partie de la construction du problème, qui subordonne l'action politique à un consensus scientifique. Or, malgré l'expertise exigeante du Giec, le diagnostic du changement climatique global ne parvient pas à entraîner une action politique globale (ou pas à un niveau suffisant). Cet échec doit peu aux sceptiques, et beaucoup à la difficulté de concilier des priorités contradictoires, aux rapports de

\footnotetext{
39 L'accusation de réductionnisme renvoie aussi aux critiques de la modélisation par des chercheurs de pays en développement dans les années 1990. Les modèles physiques omettent les conditions socioéconomiques des émissions de gaz à effet de serre (le méthane des rizières et le $\mathrm{CO}_{2}$ des voitures sont mis en équivalence). Simulant l'évolution du climat à partir d'un état de référence (1990), ils ont été accusés de naturaliser le présent, effacer le passé et globaliser le futur (Demeritt, 2001 ; Dahan et Guillemot, 2006).

40 Cette tonalité d'urgence catastrophiste se retrouve dans le manifeste "Notre génération face au jugement de l'histoire", publié dans 56 journaux de 44 pays à la veille du sommet de Copenhague de décembre 2009: "Le changement climatique résulte d'une action sur plusieurs siècles, il aura des conséquences qui dureront pour toujours, et nos chances de le mâ̂triser vont être déterminées dans les quatorze jours qui viennent. »

41 La tribune d'Allègre dans Le Monde du 22 mai 2010, mentionnée plus haut, passe en revue presque tout l'éventail des désaccords et critiques, bien au-delà de la négation de la réalité du changement climatique.
}

force entre États, aux intérêts industriels, aux besoins d'énergie des pays émergents - c'est-à-dire à la politique, à la diplomatie et à l'économie.

Ce n'est pas la confiance dans la réalité du changement climatique, mais le cadrage même du problème qui est en cause ici. La science du climat, comme l'écrit Ulrich Beck (2001) « devient de plus en plus nécessaire mais de moins en moins suffisante à l'élaboration d'une définition socialement établie de la vérité ». Le constat scientifique du changement climatique n'est pas univoque, il peut revêtir plusieurs significations selon les groupes qui se l'approprient. Les sciences du climat ne déterminent pas de politique : elles produisent suffisamment de connaissances, assorties d'incertitudes assez larges, pour justifier tout un éventail d'actions et servir d'arguments à des visions différentes (Sarewitz, 2000). Il existe donc "de multiples raisons d'être en désaccord sur le changement climatique » (Hulme, 2009), qu'il s'agisse de la gravité du problème et du rôle des enjeux climatiques (qu'on songe au casse-tête de l'attribution des événements météorologiques extrêmes au CCA) ; de l'usage et des limites des sciences du climat (avec les vifs débats, dans les communautés de climatologues, sur les prévisions régionales et décennales); de l'expertise, de son autorité (avec des propositions diverses de réformes du Giec) ; des mesures pour lutter contre le changement climatique et s'y adapter (de la croissance verte à la décroissance, de la sobriété énergétique à la géo-ingénierie, des changements de comportements individuels aux investissements publics, etc.). Le temps est sans doute venu de se détourner de l'obsession du climatoscepticisme pour déployer la diversité de ces désaccords. Il s'agirait, au lieu d'enfermer l'avenir dans une fausse alternative, de l'ouvrir à une pluralité de possibles.

\section{Références}

Aykut, S., Dahan, A., 2011. Le régime climatique avant et après Copenhague: science, politique et l'objectif de deux degrés, Natures Sciences Sociétés, 19, 144-157.

Aykut, S., Comby, J.-B., Guillemot, H., 2012. Climate change controversies in French mass media 1990-2010, Journalism Studies, 13, 2, 157-174.

Beck, U., 2001. La société du risque: sur la voie d'une autre modernité, Paris, Aubier.

Cannon, T., Müller-Mahn, D., 2010. Vulnerability, resilience and development discourses in context of climate change, Natural Hazards, 55, 3, 621-635.

Chappellaz, J., Godard, O., Huet, S., Le Treut, H., 2010. Changement climatique : les savoirs et les possibles, Montreuil, Éditions La ville brûle.

Comby, J.-B., Grossetête, M., 2012. Se montrer prévoyant: une norme sociale diversement appropriée, Sociologie, 3, 3, 251-266.

Comby, J.-B., Romanet, V., 2012. Un problème réchauffé ? Les changements climatiques dans les médias généralistes 20072011. Rapport de recherche, Institut français de presse de 
l'université Paris II, Gis «Climat, environnement et société », Paris.

Crucifix, M., 2011. La climatologie aujourd'hui, Revue des Questions Scientifiques, 182, 1, 3-32.

Dahan, A., Guillemot, H., 2006. Le changement climatique : dynamiques scientifiques, expertise, enjeux géopolitiques, Revue de Sociologie du Travail, 48, 3, 412-432.

Demeritt, D., 2001. The construction of global warming and the politics of science, Annals of the Association of American Geographers, 91, 2, 307-337.

Deser, C., Knutti, R., Solomon, S., Phillips, A., 2012. Communication of the role of natural variability in future North American climate, Nature Climate Change, 2, 11, 775-779.

Dessai, S., Hulme, M., Lempert, R., Pielke, Jr., R.A., 2009. Do we need better predictions to adapt to a changing climate?, EOS, 90, 13, 111-112.

Edwards, P., 1999. Global climate science, uncertainty and politics: Data-laden models, models-filtered data, Science as Culture, 8,4 .

Forsyth, T., 2003. Critical Political Ecology: The Politics of Environmental Science, London, Routledge.

Foucart, S., 2010. Le Populisme climatique: Claude Allègre et Cie, enquête sur les ennemis de la science, Paris, Denoël.

Funtowicz, S., Ravetz, J., 1993. Science for the post-normal age, Futures, 25, 7, 739-755.

Gieryn, T., 1983. Boundary-work and the demarcation of science from non-science: Strains and interests in professional ideologies of scientists, American Sociological Review, 48, 6, 781-795.

Godard, O., 2012. Les controverses climatiques en France : la logique du trouble, in Zaccai, E., Gemenne, F., Decroly, J.-M. (Ed.), Controverses climatiques, sciences et politiques, Les presses de SciencesPo, 117-140.

Guillemot, H., 2007. La modélisation du climat en France des années 1970 aux années 2000: histoire, pratiques, enjeux politiques. Thèse de doctorat en Histoire des sciences, EHESS, Paris.

Guillemot, H., 2010. Connections between climate simulations and observation in climate computer modeling. Scientist's practices and 'bottom-up epistemology' lessons, Studies in History and Philosophy of Modern Physics, 41, 242-252.

Gusfield, J., 1981. The Culture of Public Problems: Drinking-driving and the Symbolic Order, Chicago, Chicago University Press.

Hulme, M., 2009. Why We Disagree about Climate Change: Understanding Controversy, Inaction and Opportunity, Cambridge (UK), Cambridge University Press.

Hulme, M., 2011. Reducing the future to climate: A story of climate determinism and reductionism, Osiris, 26, 1, 245-266.

Hulme, M., Mahony, M., 2010. Climate change: What do we know about the IPCC?, Progress in Physical Geography, 34, 5, 1-14.

Jasanoff, S., 1987. Contested boundaries in policy-relevant science, Social Studies of Science, 17, 2, 195-230.

Jasanoff, S., 2010. A new climate for society, Theory, Culture $\mathcal{E}$ Society, 27, 2-3, 233-253.

Jasanoff, S., Wynne, B., 1998. Science and decision-making, in Rayner, S., Malone, E.L. (Eds), Human choice and climate change, 1, Columbus (OH), Battelle Press, 1-87.

Knorr Cetina, K., 1999. Epistemic Cultures: How the Sciences Make Knowledge, Cambridge (MA), Harvard University Press.

Reçu le 24 décembre 2012. Accepté le 5 avril 2014.
Lahsen, M., 2008. Experiences of modernity in the greenhouse: A cultural analysis of a physicist 'trio' supporting the backlash against global warming, Global Environmental Change, 18, 204-219.

Latour, B., 1991. Nous n'avons jamais été modernes, Paris, La Découverte.

Latour, B., 2012. Enquête sur les modes d'existence: une anthropologie des Modernes, Paris, La Découverte.

Lecourt, D., 1993. Contre la peur suivi de Critique de l'appel de Heidelberg, Coll. Pluriel, Paris, Hachette.

Lemieux, C., 2007. À quoi sert l'analyse des controverses ?, Mil Neuf Cent, 1, 25, 29-43.

Lévy-Leblond, J.-M., 1996. La Pierre de touche, Paris, Gallimard.

McCright, A.M., Dunlap, R.E., 2000. Challenging global warming as a social problem: An analysis of the conservative movement's counter-claims, Social Problems, $47,4,227-48$.

Mercer, J., 2010. Disaster risk reduction or climate change adaptation: Are we reinventing the Wheel?, Journal of International Development, 22, 247-264.

Miller, C., 2004. Climate science and the making of a global political order, in Jasanoff, S. (Ed.), States of Knowledge: The CoProduction of Science and Social Order, London, Routledge.

Millier, C., 2011. Heidelberg, vingt ans après, Natures Sciences Sociétés, 3, 19.

Norton, S., Suppe, F., 2001. Why atmospheric modeling is good science, in Miller, C., Edwards, P. (Ed.), Changing the Atmosphere: Expert Knowledge and Environmental Governance, Cambridge (MA), MIT Press.

Oreskes, N., Conway, E., 2010. Merchands of doubt: How a Handful of Scientists Obscured the Truth on Issues from Tobacco Smoke to Global Warming, London, Bloomsbury Press.

Pestre, D., 2006. Introduction aux Science Studies, Paris, La Découverte.

Pielke, Jr., R.A., 2002. Policy, politics and perspective, Nature, 416,368 .

Sarewitz, D., 2000. Science and environmental policy: An excess of objectivity, in Frodeman, R. (Ed.), Earth Matters: The Earth Sciences, Philosophy, and the Claims of Community, Prentice Hall, Upper Saddle River (NJ), 79-98.

Sarewitz, D., 2004. How science makes environmental controversies worse, Environmental Science and Policy, 7, 385-403.

Shackley, S., Risbey, J., Stone, P., Wynne, B., 1999. Adjusting to policy expectations in climate change modeling: An interdisciplinary study of flux adjustments in coupled atmosphere-ocean general circulation models, Climatic Change, 43, 413-454.

Stengers, I., 1993. L'Invention des sciences modernes, Paris, La Découverte.

Swyngedouw, E., 2010. Apocalypse forever? Post-political populism and the spectre of climate change, Theory, Culture $\mathcal{E}$ Society, 27, 213-232.

Trenberth, K., 2010. More knowledge, less certainty, Nature Reports Climate Change, 20-21.

Van der Sluijs, J., Van Eijndhoven, J., Shackley, S., Wynne, B., 1998. Anchoring devices in science for policy: The case of consensus around climate sensitivity, Social Studies of Science, 28, 2.

Wynne, B., 2010. When doubt becomes a weapon, Nature, 466, 441-442. 